\title{
Puerarin Attenuates Cerebral Damage by Improving Cerebral Microcirculation in Spontaneously Hypertensive Rats
}

\author{
Xu-Dong Wu, ${ }^{1}$ Chen Wang, ${ }^{2,3}$ Zhen-Ying Zhang, ${ }^{2,3}$ Yan Fu, \\ Feng-Ying Liu, ${ }^{2,3}$ and Xiu-Hua Liu ${ }^{2,3}$ \\ ${ }^{1}$ Out-Patient Department, Chinese PLA General Hospital, Beijing 100853, China \\ ${ }^{2}$ Department of Pathophysiology, Chinese PLA General Hospital, Beijing 100853, China \\ ${ }^{3}$ State Key Laboratory of Kidney Disease, Chinese PLA General Hospital, 2011DAV00088, Beijing 100853, China \\ Correspondence should be addressed to Xiu-Hua Liu; xiuhualiu98@163.com
}

Received 26 October 2013; Accepted 7 December 2013; Published 13 February 2014

Academic Editor: Syed Mohammed Basheeruddin Asdaq

Copyright (C) $2014 \mathrm{Xu}$-Dong Wu et al. This is an open access article distributed under the Creative Commons Attribution License, which permits unrestricted use, distribution, and reproduction in any medium, provided the original work is properly cited.

\begin{abstract}
Puerariae Lobatae Radix (Gegen in Chinese) is the dried root of Pueraria lobata, a semiwoody, perennial, and leguminous vine native to China. Puerarin is one of the effective components of isoflavones isolated from the root of Pueraria lobata. Previous studies showed that extracts derived from the root of Pueraria lobata possessed antihypertensive effect. Our study is to investigate whether puerarin contributes to prevention of stroke by improving cerebral microcirculation in rats. Materials and Methods. Video microscopy and laser Doppler perfusion imaging on the pia mater were used to measure the diameter of microvessel and blood perfusion in 12-week old spontaneously hypertensive rats (SHRs) and age-matched normotensive WKY rats. Histological alterations were observed by hematoxylin and eosin staining, and microvessel density in cerebral tissue was measured by immunohistochemical analysis with anti-Factor VIII antibody. Cell proliferation was detected by $\left[{ }^{3} \mathrm{H}\right]-\mathrm{TdR}$ incorporation, and activities of p42/44 mitogen activated protein kinases (p42/44 MAPKs) were detected by western blot analysis in cultured cerebral microvascular endothelial cells (MECs). Results. Intravenous injection of puerarin relaxed arterioles and increased the blood flow perfusion in the pia mater in SHRs. Puerarin treatment for 14 days reduced the blood pressure to a normal level in SHRs $(P<0.05)$ and increased the arteriole diameter in the pia mater significantly as compared with vehicle treatment. Arteriole remodeling, edema, and ischemia in cerebral tissue were attenuated in puerarin-treated SHRs. Microvessel density in cerebral tissue was greater with puerarin than with vehicle treatment. Puerarin-treated MECs showed greater proliferation and p42/44 MAPKs activities than vehicle treatment. Conclusions. Puerarin possesses effects of antihypertension and stroke prevention by improved microcirculation in SHRs, which results from the increase in cerebral blood perfusion both by arteriole relaxation and p42/44 MAPKs-mediated angiogenesis.
\end{abstract}

\section{Introduction}

Hypertension is the key risk factor for stroke, the second leading cause of death in China, and is characterized by increased peripheral vascular resistance and microvascular abnormalities [1, 2]. When antihypertensive drugs reduce systemic blood pressure, the microvessel dysfunction contributes to decreased perfusion pressure in the brain [3]. The decreased perfusion pressure impairs blood perfusion in cerebral tissue and might lead to stroke. Hence, identifying novel therapies for more effective and safer antihypertensive drugs with microcirculation improvement in patients with imminent stroke is urgently needed.
Pueraria Lobata Radix (Gegen in Chinese) is the dried root of Pueraria lobata (Wild.) Ohwi, a semiwoody, perennial, and leguminous vine native to China, which has been used as an herbal medicine for the treatment of fever, diarrhea, headache, and cardiovascular diseases for more than 2000 years [4]. Puerarin is the first identified effective component from the root of Pueraria Lobata Radix [5] and has been used as the marker compound in herb quality evaluation in the Chinese Pharmacopoeia [6]. The other major ingredients of Pueraria Lobata Radix include flavonoid-including daidzin, daidzein, daidzein-4-7-glucoside, puerarin-7-xyloside, 4-6O-acetyldaidzin, and alkaloid [7]. Total flavonoids of pueraria 
reduced blood pressure in a dose-dependent manner in spontaneously hypertensive rats (SHRs) [8], which indicates that pueraria reduces blood pressure and morbidity of stroke.

Puerarin injection has been widely employed in China for the treatment of acute ischemic stroke [4]. However, few reports are available on the mechanisms underlying stroke prevention of Puerarin. We hypothesized that puerarin contributes to the prevention of stroke by improving cerebral microcirculation and protecting brain against ischemia damage. We investigated the effects of puerarin both on pia mater microcirculation in SHRs and in cultured cerebral microvascular endothelial cells (MECs) by investigating the proliferation effects and its signaling pathway. We aimed to explain the mechanism by which puerarin contributes to prevention of stroke through improving cerebral microcirculation.

\section{Material and Methods}

\subsection{Animals and Chemicals}

2.1.1. Animals. Twelve-week-old SHRs and age-matched normotensive Wistar-Kyoto (WKY) rats were housed 4 per cage, at $23^{\circ} \mathrm{C}$, with luminosity cycles of $12 \mathrm{~h}$ light $/ 12 \mathrm{~h}$ dark, fed regular rat chow, and allowed free access to water. The rats were provided by the Beijing Institute of Experimental Animals. All procedures were performed in accordance with the Guide for the Care and Use of Laboratory Animals published by the US National Institutes of Health (NIH Publication number 85-23, revised 1996) and approved by the local animal care and use committee.

2.1.2. Chemicals. Puerarin was purchased from the Beijing Union Pharmaceutical Factory (Beijing, China). M199 medium was from Gibco Co. (Carlsbad, CA, USA). Endothelial cell growth supplement (ECGS), collagenase I, tetramethyl ethylene diamine (TEMED), phenylmethyl sulfonylfluoride (PMSF), sodium dodecyl sulphate (SDS), $\beta$-glycerophosphate, sodium orthovanadate, leupeptin, DTT, and anti-Factor VIII antibody were from Sigma Chemical Co. (St. Louis, MO, USA). Antibodies for total and phosphop42/p44-MAPKs were from Santa Cruz Biotechnology. PD98059 was from Calbiochem. Prestained protein marker was from Bio-Rad Co. Antibodies specific for nonphosphorylated and phospho-specific p42/p44-MAPKs and avidinbiotin-peroxides complex (ABC) kit for immunohistochemistry were from Santa Cruz Biotechnology (Santa Cruz, CA, USA). Low-molecular-weight calibration kit for SDS electrophoresis was from Amersham Biosciences Co. (Buckinghamshire, UK); cocktail tablets were from Roche Co. (Basel, Switzerland).

\subsection{Microscopy Monitoring of Pia Mater Microcirculation.} After a 5-day acclimatization, a $4 \mathrm{~mm}$ diameter cranial window was drilled, and cerebral dura mater was separated in rats under anesthesia with pentobarbital sodium $(30 \mathrm{mg} / \mathrm{kg})$ as described previously [9]; the microcirculation of pia mater was monitored by use of a biomicroscope (BH-2, Olympus, Japan) equipped with a video camera (BY-110, JVC, Japan) and a computer. Blood flow perfusion of the cerebral pia mater was detected by use of Laser Doppler Perfusion Imager (LISCAPIMII, Sweden). After baseline monitoring, rats received puerarin $(100 \mathrm{mg} / \mathrm{kg})$ in $20 \%$ propanediol by tail-vein injection, and microcirculation and blood perfusion were monitored at $3,5,10,15,20,30$, and 40 min after puerarin injection.

2.3. Hemodynamic Measurements. Twelve-week-old male SHRs and age-matched normotensive WKY rats were housed and fed for 1 week and randomly divided into 7 groups ( $n=10)$ for treatment: puerarin-treated SHRs and WKY rats, intraperitoneal injection of $100 \mathrm{mg} / \mathrm{kg}$ puerarin in $0.3 \mathrm{~mL}$ of vehicle ( $20 \%$ propanediol) every day for 14 days; vehicletreated SHRs and WKY, the same volume (20\%) of propanediol injected every day for 14 days; age-matched control SHRs and WKY rats, rats housed and fed routinely for 14 days; and nimodipine, SHRs receiving $30 \mathrm{mg} / \mathrm{kg}$ nimodipine by gastrogavage every day for 14 days. All rats were anesthetized with $20 \%$ urethane $(10 \mathrm{~mL} / \mathrm{kg})$ by intraperitoneal injection $1 \mathrm{hr}$ after the last treatment (or the same time in controls). Microcirculation and blood flow perfusion of cerebral pia mater were monitored as described above. Then mean arterial pressure (MAP) and heart rate were measured for consecutive $3 \mathrm{~min}$ in anesthetized rats with the right carotid artery cannulated and connected to a pressure transducer in line to a Grass polygraph (SMUP-PCI).

\subsection{Immunohistochemical and Histological Staining of Cere-} bral Tissue. At the end of the experiment and within $3 \mathrm{~min}$ of sacrifice, brains were removed from rats, 2 coronal slices were made at 5 and $7 \mathrm{~mm}$ from the frontal pole, and brain slices were immersed in $10 \%$ phosphate-buffered formalin, routinely processed, and embedded in paraffin. Serial sections $(6 \mu \mathrm{m})$ were stained by hematoxylin and eosin for morphological assessment or dipped in cold acetone for $10 \mathrm{~min}$ for immunohistochemical staining [10]. To reduce nonspecific reactivity, sections were preincubated with $0.3 \%$ hydrogen peroxide and normal goat serum. Sections were incubated at $4^{\circ} \mathrm{C}$ overnight with rabbit antihuman Factor VIII-related antigen (1:200 dilution), a marker of endothelial cells, and then for $1 \mathrm{hr}$ at $37^{\circ} \mathrm{C}$ with goat anti-rabbit immunoglobulin (1:500 dilution). Antibody-biotin conjugate was detected with use of a $\mathrm{DAB}$ (3,3N-Diaminobenzidine Tertrahydrochloride) kit according to the manufacturer's instructions. Microvessels were identified as being between 10 and $25 \mu \mathrm{m}$. We randomly chose 10 fields at 50x magnification for microvessel counting; the number of microvessels is presented as number per $\mathrm{mm}^{2}$.

2.5. Culture of MECs. The MECs from SHRs and WKY rat pallium were cultured as described [11] with modification. Briefly, brain cortex was obtained from urethaneanesthetized SHRs or WKY rats. The pallium was fully minced, and cerebral microvessels were collected by filtrating the homogenate in medium 199 (M199) through 300 and $60 \mu \mathrm{m}$ nylon mesh screens consecutively. The filtrate was collected and suspended in M199 medium containing $2 \mathrm{mmol} / \mathrm{L}$ 
TABLE 1: Effect of puerarin on mean arterial blood pressure (MAP), blood perfusion, and microvessel diameter in Wistar-Kyoto (WKY) rats and spontaneously hypertensive rats (SHRs).

\begin{tabular}{|c|c|c|c|c|c|}
\hline \multirow{2}{*}{ Groups } & \multirow{2}{*}{$\begin{array}{l}\text { Heart rate } \\
\text { (beat } / \mathrm{min})\end{array}$} & \multirow{2}{*}{$\begin{array}{c}\text { MAP } \\
(\mathrm{mmHg})\end{array}$} & \multirow{2}{*}{$\begin{array}{l}\text { Blood perfusion } \\
\text { (PU) }\end{array}$} & \multicolumn{2}{|c|}{ Microvessel diameter $(\mu \mathrm{m})$} \\
\hline & & & & Arterioles & Venule \\
\hline \multicolumn{6}{|l|}{ WKY } \\
\hline Control & $389 \pm 21$ & $83.72 \pm 7.82$ & $4.89 \pm 1.09$ & $42.64 \pm 10.43$ & $44.72 \pm 9.51$ \\
\hline Vehicle & $387 \pm 12$ & $90.73 \pm 19.67$ & $3.33 \pm 0.89$ & $44.78 \pm 6.04$ & $43.66 \pm 4.51$ \\
\hline Puerarin & $391 \pm 28$ & $91.51 \pm 13.58$ & $4.16 \pm 0.18$ & $47.8 \pm 10.91$ & $43.78 \pm 4.04$ \\
\hline \multicolumn{6}{|l|}{ SHRs } \\
\hline Control & $401 \pm 26$ & $99.80 \pm 16.16^{*}$ & $4.43 \pm 0.77$ & $42.82 \pm 7.69$ & $33.29 \pm 8.24$ \\
\hline Vehicle & $398 \pm 38$ & $105.92 \pm 22.61$ & $3.84 \pm 0.82$ & $42.84 \pm 6.83$ & $46.08 \pm 15.67$ \\
\hline Puerarin & $382 \pm 21$ & $85.56 \pm 15.62^{\#}$ & $3.83 \pm 0.89$ & $55.20 \pm 15.12^{\#}$ & $47.20 \pm 15.13$ \\
\hline Nimodipine & $378 \pm 35$ & $113.38 \pm 10.10^{\#}$ & $4.76 \pm 0.58$ & $49.94 \pm 12.74^{\#}$ & $41.74 \pm 7.09$ \\
\hline
\end{tabular}

Data are mean \pm SD, $n=7$. PU: perfusion unit. ${ }^{*} P<0.05$ compared with WKY control group; ${ }^{*} P<0.05$ compared with SHRs vehicle.

L-glutamine, $100 \mathrm{U} / \mathrm{mL}$ penicillin, $100 \mu \mathrm{g} / \mathrm{mL}$ streptomycin, $25 \%$ fetal bovine serum (FBS), $40 \mathrm{U} / \mathrm{mL}$ heparin, and $100 \mu \mathrm{g} / \mathrm{mL}$ ECGS. The suspension was cultured in a humidified atmosphere with $5 \% \mathrm{CO}_{2}$ at $37^{\circ} \mathrm{C}$. MECs were identified by their morphology under phase-contrast microscopy and by Factor VIII-related antigen. All individual batches of MECs were prepared from a single rat pallium. All experiments were performed on confluent quiescent MECs at the third or fourth passage and were exposed to serum-depleted medium ( $0.1 \%$ FBS) for $12 \mathrm{hr}$ before the experiment.

2.6. Assessment of Cell Proliferation by $\left[{ }^{3} H\right]-T d R$ Incorporation. Cells were plated in 24 -well plates at $10^{4}$ cells/well in M199 supplemented with 10\% FBS and $100 \mu \mathrm{g} / \mathrm{mL}$ ECGS. After $24 \mathrm{hr}$ incubation, MECs were rinsed with phosphatebuffered saline and incubated in $0.1 \%$ FBS and ECGSdeprived M199 for $12 \mathrm{~h}$. Then, MECs from SHRs and WKY rats were randomly divided into experimental groups for treatment: puerarin, incubated with puerarin $(25,50$, and $100 \mathrm{ng} / \mathrm{L})$ for $24 \mathrm{~h}$; control, incubated with vehicle $(20 \%$ propanediol) for $24 \mathrm{hr}$; FBS, MECs from SHRs incubated with $10 \%$ FBS for $24 \mathrm{hr}$; PD98059+puerarin, pretreated with PD98059 (MEK-1/2 inhibitor, $50 \mu \mathrm{mol} / \mathrm{L}$ ) for $10 \mathrm{~min}$ before puerarin $(100 \mathrm{ng} / \mathrm{L})$ treatment; and $\mathrm{BDM}+$ puerarin, pretreated with an activator of protein phosphatase, 2,3butanedione monoxide (BDM, $20 \mathrm{mmol} / \mathrm{L}) 10 \mathrm{~min}$ before puerarin $(100 \mathrm{ng})$. Then cultures were incubated with $1 \mu \mathrm{Ci}$ $\left[{ }^{3} \mathrm{H}\right]-\mathrm{TdR}$ (Amersham, USA) for $12 \mathrm{hr}$ [12]. At the end of incubation, cells were washed twice with $4^{\circ} \mathrm{C}$ PBS and detached from wells by trypsinization. Detached MECs were harvested onto glass fiber filter paper by use of a mini-MASH II microharvesting device (Whittaker MA Bioproducts, Walkesville, MD), and $\left[{ }^{3} \mathrm{H}\right]$-TdR incorporated into MECs was determined by use of a liquid scintillation counter (Beckman LS 6500).

2.7. Western Blot Analysis of ERKs. MECs were rinsed and homogenized in ice-cold extraction buffer containing (in $\mathrm{mmol} / \mathrm{L}$ ) Hepes 20 ( $\mathrm{pH} 7.7), \mathrm{MgCl}_{2} 2.5$, EDTA 0.1, $\beta$ glycerophosphate 20 , DTT 0.5 , sodium orthovanadate 0.1 ,
$\mathrm{NaCl} 75$, Leupeptin $4(\mu \mathrm{g} / \mathrm{mL})$, PMSF $20(\mu \mathrm{g} / \mathrm{mL})$, and triton $\mathrm{X}-1000.05 \%(\mathrm{v} / \mathrm{v})$. The homogenate was incubated and centrifuged at $4^{\circ} \mathrm{C}$. Protein concentration in the detergent soluble supernatant was determined by use of a bicinchoninic acid protein assay kit according to the manufacturer's protocol. The supernatant was mixed with Laemmli buffer and heated for $5 \mathrm{~min}$ at $95^{\circ} \mathrm{C}$. Soluble extracts $(50 \mu \mathrm{g})$ were loaded per lane and separated by SDS-PAGE and then electrophoretically transferred to a polyvinylidene difluoride filter membrane $(0.45 \mu \mathrm{m})$ and blocked with $10 \%$ nonfat dry milk. The blots were then incubated with the antibodies against total and phospho-p42/p44-MAPKs at 1:1000 dilution in TBS$\mathrm{T}$ and then horseradish peroxidase-conjugated anti-rabbit IgGin TBS-T [13]. Antibody-antigen complexes were visualized by enhanced chemiluminescence. Membranes were exposed to X-ray film for $30 \mathrm{~s}$ to $2 \mathrm{~min}$. The staining was quantified by scanning the films, and the band density was determined with use of Image-Pro software.

2.8. Statistical Analysis. Data is expressed as mean $\pm \mathrm{SD}$ from at least 3 (immunohistochemical, immunoblotting, and histological staining) or 7 (measurement of hemodynamics and microcirculation) independent experiments. Differences in means between groups were tested by one-way ANOVA. $P<0.05$ was considered statistically significant. All statistical analyses involved use of SPSS v12.0 (SPSS Inc., Chicago, IL).

\section{Results}

3.1. Puerarin Lowered Blood Pressure in SHRs. Hemodynamic data is shown in Table 1 . The groups did not differ in heart rate $(P>0.05)$. The MAP was higher for SHRs than for WKY rats treated with vehicle $(P<0.05)$, with no significant differences in MAP for these rats than their controls $(P>$ $0.05)$, respectively, which indicates that vehicle injection did not affect blood pressure of rats. WKY rats treated with or without puerarin did not differ in MAP $(P>0.05)$, which suggests that puerarin did not affect blood pressure in normotensive WKY rats. However, as compared with vehicletreated SHRs, puerarin-treated SHRs showed significantly lower MAP, by $19.2 \%(P<0.05)$, which was similar to that for 


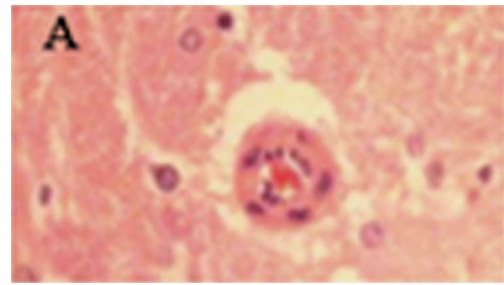

(a)

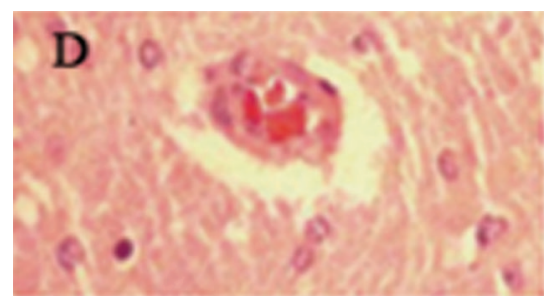

(d)

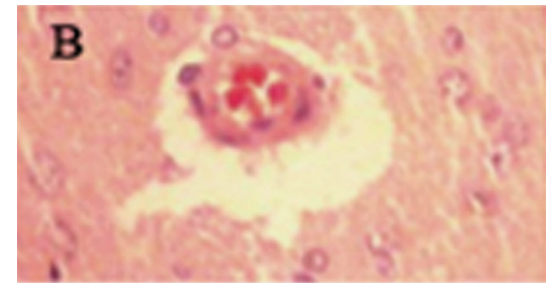

(b)

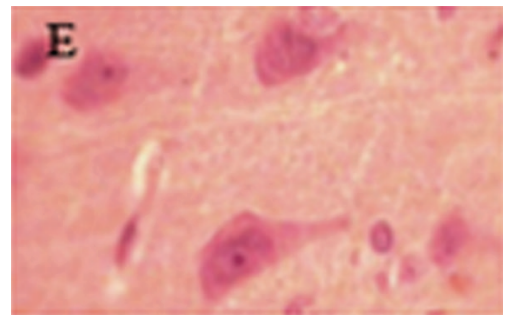

(e)

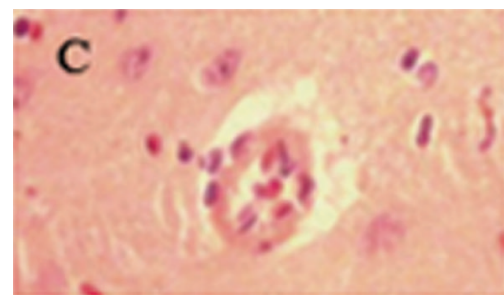

(c)

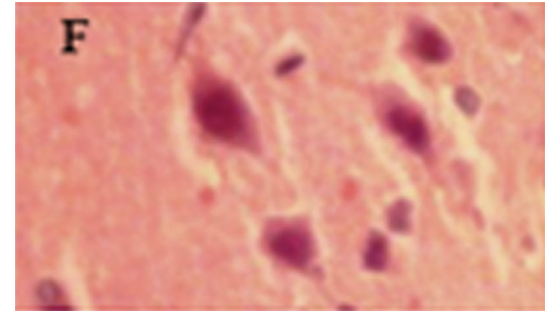

(f)

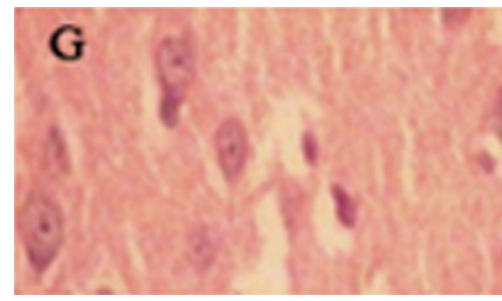

(g)

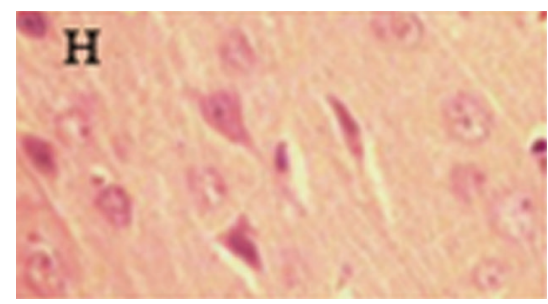

(h)

FIGURE 1: Effect of puerarin on brain microvascular remodeling in rats. (a)-(d) Brain microvascular remodeling. (a) Wistar-Kyoto (WKY) rats, (b) spontaneously hypertensive rats (SHRs), and (c) SHRs receiving puerarin (100 $\mathrm{mg} / \mathrm{kg}$ puerarin) for 14 days by intraperitoneal injection. (d) SHRs receiving nimodipine $(30 \mathrm{mg} / \mathrm{kg})$ by gastrogavage for 14 days (serial sections $(6 \mu \mathrm{m})$ were stained by hematoxylin and eosin $((\mathrm{h})-(\mathrm{e}))$ as described in text, $\times 100)$.

WKY control rats $(P>0.05)$, which indicates that puerarin had an antihypertensive effect in SHRs.

3.2. Puerarin Attenuated Cerebral Injury in SHRs. H-E staining revealed normal structure in neurons and the arteriole wall in WKY rats but remodeling and hyaline degeneration in the arteriole wall (Figures 1(a)-1(d)) and regional ischemic neurodegeneration, edema, and neuron karyopyknosis (Figures $1(\mathrm{e})-1(\mathrm{~h})$ ) in SHRs, which regularly expanded to the basal ganglia and the neocortex. Puerarin treatment for 14 days attenuated these morphological alterations as compared with vehicle treatment.

3.3. Puerarin Treatment Increased Microvessel Density in Cerebral Tissue. Immunohistochemistry revealed microvessel density in cerebral tissue significantly increased in SHRs with puerarin treatment for 14 days as compared with propanediol-treated and control SHRs (Figure 2).

3.4. Puerarin Treatment Improved Pia Mater Microcirculation. Puerarin injection induced significant arteriole dilation in pia mater of SHRs and WKY rats at 3 min after injection, as compared with baseline values $(P<0.05)$ (Table 2$)$. The arteriole relaxation in normotensive WKY rats peaked at

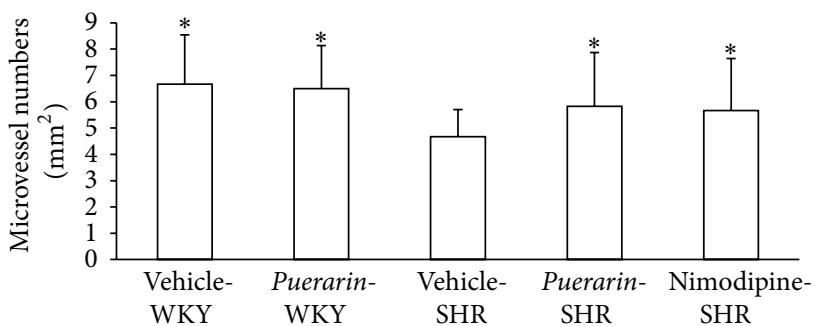

FIGURE 2: Effect of puerarin on brain microvessel density in rats. ${ }^{*} P<0.05$ compared with SHRs receiving vehicle $(20 \%$ propanediol).

$10 \mathrm{~min}$ and was sustained to $40 \mathrm{~min}$ after puerarin injection $(P<0.05)$. In SHRs, the arteriole relaxation peaked at $30 \mathrm{~min}$, when the internal arteriole diameter increased by $26 \%$ as compared with baseline $(P<0.05)$. Moreover, SHRs and WKY rats did not differ in extent of arteriole relaxation. The internal diameter of the venule in both groups remained unchanged after puerarin treatment (Figures 3(a) and 3(b)).

Laser Doppler perfusion revealed that the baseline level of blood perfusion of pia mater in WKY rats was significantly higher than that in SHRs at baseline $(P<0.05)$ (Table 3$)$. In WKY rats, puerarin injection increased blood perfusion in 
TABLE 2: Effect of puerarin on internal diameter of pia mater microvessels in rats.

\begin{tabular}{|c|c|c|c|c|c|c|c|c|}
\hline \multirow{2}{*}{ Groups } & \multirow{2}{*}{ Baseline $(\mu \mathrm{m})$} & \multicolumn{7}{|c|}{ After puerarin injection $(\mu \mathrm{m})$} \\
\hline & & $3 \mathrm{~min}$ & $5 \mathrm{~min}$ & $10 \mathrm{~min}$ & $15 \mathrm{~min}$ & $20 \mathrm{~min}$ & $30 \mathrm{~min}$ & $40 \mathrm{~min}$ \\
\hline WKY & $41.24 \pm 10.97$ & $49.76 \pm 9.32$ & $50.11 \pm 12.78$ & $52.12 \pm 12.41^{*}$ & $52.08 \pm 12.10^{*}$ & $52.23 \pm 14.09^{*}$ & $52.02 \pm 13.72^{*}$ & $51.47 \pm 17.25^{*}$ \\
\hline SHRs & $44.43 \pm 7.90$ & $46.13 \pm 7.29$ & $51.89 \pm 7.15^{*}$ & $51.69 \pm 11.30^{*}$ & $54.16 \pm 11.41^{*}$ & $55.16 \pm 10.86^{*}$ & $55.95 \pm 9.892$ & $55.70 \pm 12.09$ \\
\hline
\end{tabular}

Data are mean $\pm \mathrm{SD}, n=7 .{ }^{*} P<0.05$ compared with baseline level.

TABLE 3: Effect of puerarin on blood perfusion in rat pia mater.

\begin{tabular}{|c|c|c|c|c|c|}
\hline \multirow{2}{*}{ Groups } & \multirow{2}{*}{ Baseline (PU) } & \multicolumn{4}{|c|}{ After puerarin injection (PU) } \\
\hline & & $10 \mathrm{~min}$ & $20 \mathrm{~min}$ & $30 \mathrm{~min}$ & $40 \mathrm{~min}$ \\
\hline WKY & $5.89 \pm 1.10$ & $6.96 \pm 1.71^{* \#}$ & $6.58 \pm 1.69$ & $6.40 \pm 1.53$ & $6.12 \pm 1.47$ \\
\hline SHRs & $4.94 \pm 0.45^{*}$ & $6.02 \pm 1.23^{* \#}$ & $5.69 \pm 1.05^{*}$ & $5.19 \pm 1.05^{*}$ & $4.94 \pm 0.57^{*}$ \\
\hline
\end{tabular}

Data are mean \pm SD, $n=7$. PU: perfusion unit. ${ }^{*} P<0.05$ compared with WKY group; ${ }^{*} P<0.05$ compared with SHRs control group.

cerebral pia mater by $18 \%(P<0.05)$ at $10 \mathrm{~min}$ after injection as compared with baseline and decreased to the baseline level at $40 \mathrm{~min}$ after injection. In SHRs, blood perfusion was increased by $22 \%(P<0.05)$ and $15 \%(P<0.05)$ at 10 and $20 \mathrm{~min}$, respectively, after puerarin injection as compared with baseline and was reduced to the baseline level at $40 \mathrm{~min}$ after puerarin injection $(P>0.05)$.

Results from intravital microscopy revealed no significant change in diameter of microvessels after 14-day vehicle treatment in both SHRs and WKY rats as compared with controls, which indicates that propanediol had no effect on microvascular tension in pia mater in SHRs and WKY rats (Table 1). However, puerarin treatment for 14 days was associated with a significant increase in internal diameter of arterioles in SHRs. Compared with vehicle-treated and SHRs controls, the arteriole diameter with puerarin treatment in SHRs was increased by $29 \%$ and $28.8 \%(P<0.05)$, respectively (Table 1). Puerarin-induced arteriole relaxation was greater than that with nimodipine-treated relaxation. However, laser Doppler perfusion imaging showed that both puerarin and nimodipine did not change the blood flow perfusion in cerebral pia mater in all groups (Figures 3(c)$3(\mathrm{e}))$.

3.5. Puerarin Treatment Induced MECs Proliferation. SHRs and WKY control rats did not differ in $\left[{ }^{3} \mathrm{H}\right]$-TdR incorporation in MECs at $24 \mathrm{~h}(P>0.05)$ (Figure 4(a)). However, compared with vehicle treatment, puerarin treatment (50 and $100 \mathrm{ng} / \mathrm{L}$ ) induced a $74.1 \%$ and $96.5 \%$ increase, respectively, in $\left[{ }^{3} \mathrm{H}\right]-\mathrm{TdR}$ incorporation $(P>0.05)$ in SHRs. $\left[{ }^{3} \mathrm{H}\right]-\mathrm{TdR}$ incorporation with $10 \%$ FBS was increased by $103.9 \%$ as compared with SHRs control $(P<0.05)$, which was similar to puerarin, $100 \mathrm{ng}(P>0.05)$. Pretreatment with $P D 98059$, an inhibitor of the MAPK kinase MEK-1/2 (upstream activators of $\mathrm{p} 42 / 44$ MAPKs), or BDM (an activator of protein phosphatase) abolished puerarin-induced proliferation in MECs.

3.6. Puerarin Treatment Activated p42/44 MAPKs in MECs. The activity of $\mathrm{p} 42 / 44$ MAPKs was detected by western blot analysis with an antibody against phospho-Tyr-204. Treatment with puerarin $(25,50,100 \mathrm{ng} / \mathrm{L})$ resulted in $25.0 \%$,
$66.7 \%$, and $75.0 \%$ increase, respectively, in phosphorylation of the p42 MAPK isoform in MECs and $17.8 \%, 60.2 \%$, and $62.7 \%$ increase in phosphorylation of the $\mathrm{p} 44 \mathrm{MAPK}$ isoform, as compared with control treatment $(P<0.05)$ (Figures $4(\mathrm{~b})$ and 4(c)). The activity of p42- and p44-MAPK with $100 \mathrm{ng}$ puerarin was similar to that with FBS $(P>0.05)$. PD98059 or BDM abolished the activation of $\mathrm{p} 42 / 44$ MAPKs induced by puerarin (Figures 4(b) and 4(c)).

\section{Discussion}

Hypertension induces spasm, stiffening, and remodeling of resistance vessels, which results in autoregulatory dysfunction in brain arterioles [3]. Along with the elevation of systemic blood pressure, damaged vessels undergo hyperperfusion, which leads to hyperemia, edema, and hemorrhage in cerebral tissue. However, decreased blood pressure caused by antihypertensive drugs may cause hypoperfusion and induce cerebral damage and even stroke. It is urgently needed to identify novel therapies for effective antihypertension with microcirculation improvement in patients with imminent stroke. Puerarin is one of the effective ingredients of pueraria lobata, a traditional Chinese medicine, with effects on reducing blood pressure and plasma rennin activities [14] and downregulating mRNA expressions of angiotensin II type I receptor (AT1) and angiotensin-converting enzyme 2 (ACE2) in myocardium in SHRs [4]. In the present study, puerarin reduced the elevated blood pressure and prevented arteriole remodeling, edema, and ischemia in cerebral tissue in SHRs. Our finding suggested that a 14-day treatment with puerarin protected cerebral microcirculation along with the reduction of systemic blood pressure in SHRs, which agrees with other findings demonstrating that flavonoids of pueraria reduced blood pressure in SHRs and prevented the tendency to stroke in stroke-prone SHRs [8].

Puerarin injection has been widely employed in China for the treatment of acute ischemic stroke. Evidence has illustrated that puerarin confers neuroprotective action through antiapoptosis in cultured hippocampal neurons [15] and cerebral vasodilation, which reduced cerebral cortical blood flow and enhanced anterior and middle cerebral artery blood 


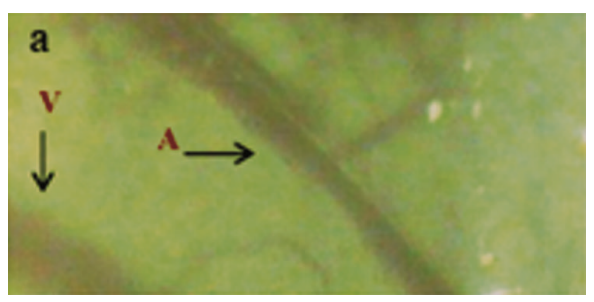

(a)

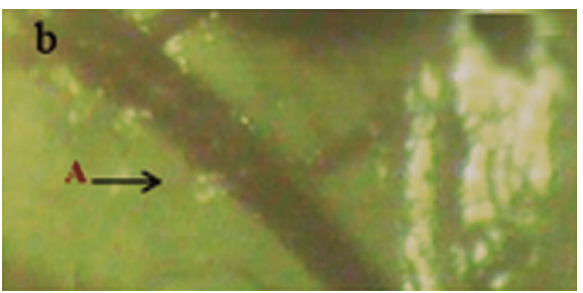

(b)

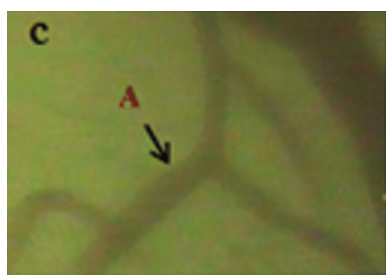

(c)

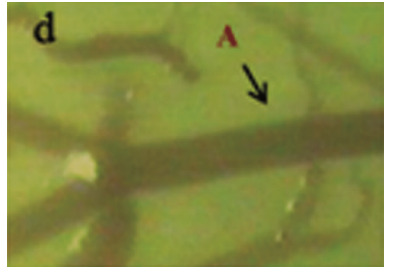

(d)

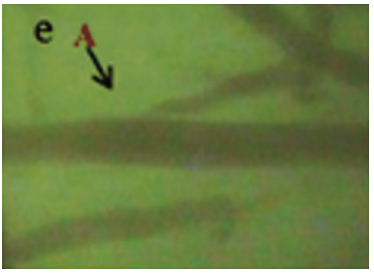

(e)

FIGURE 3: Effect of puerarin on microvessel diameter in pia mater of SHRs. (a) Before puerarin treatment. (b) 10 min after puerarin treatment. (c) SHRs control group. (d) SHRs receiving puerarin for 14 days. (e) SHRs receiving nimodipine for 14 days. A: arteriole; V: venule.

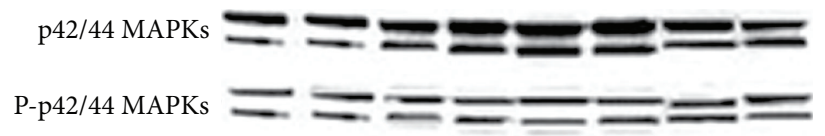

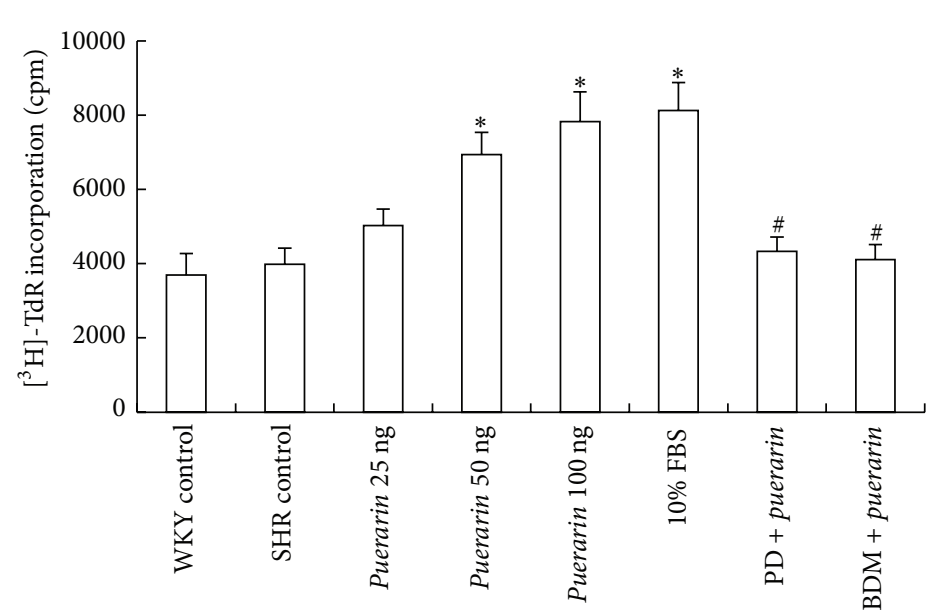

(a)

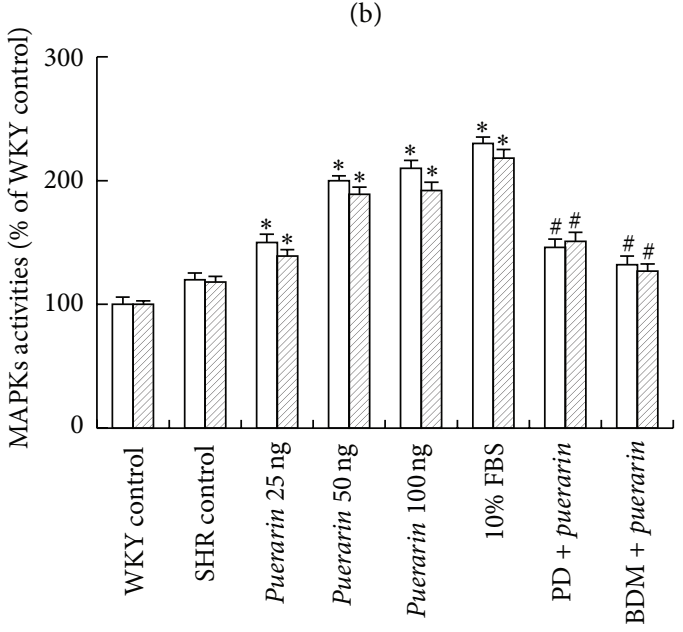

口 $\mathrm{p} 42 \mathrm{MAPK}$

$\square$ P44 MAPK

(c)

FIGURE 4: Effect of puerarin on proliferation and activity of p42/44 mitogen-activated protein kinases (MAPKs) in microvascular endothelial cells (MECs). (a) MECs proliferation detected by $\left[{ }^{3} \mathrm{H}\right]$-TdR incorporation. (b) Western blot analysis of phosphorylation of p42/44 MAPKs in MECs (upper panel). Equal protein loading was verified by reblotting with antitotal p42/44 MAPK antibody (lower panel). (c) Densitometry of the immunoblots shown in (a). Data are mean $\pm \mathrm{SD},{ }^{*} P<0.05$ compared with SHRs control, ${ }^{\#} P<0.05$ compared with puerarin treatment [100 ng], and $n=3$.

flow [16]. Our data showed that puerarin-induced cerebralprotective effects in SHRs were associated with improvement of pia mater microcirculation, as assayed by measurement of diameter of microvessels by video microscopy, blood perfusion by laser Doppler imaging, and angiogenesis by immunohistochemical staining. Puerarin injection relaxed arterioles and increased blood perfusion significantly in rat pia mater in both SHRs and normotensive WKY rats. Puerarin treatment for 14 days reduced the blood pressure, which was accompanied by a significant increase in arteriole diameter in pia mater and a notable alleviation of microvessel remodeling in brain characterized by thinness in arteriole 
tunica media and increase in brain microvessel density. Thus, puerarin improved microcirculation by inducing angiogenesis and improving arteriole tensility and elasticity. In vitro experiments with MECs showed an increase in proliferation $24 \mathrm{~h}$ after puerarin treatment. These findings suggest that puerarin may have a cerebral-protective effect along with antihypertension by improving the structure and function of microvessels and hence increasing blood perfusion in cerebral tissue.

The intracellular signaling mechanism that mediates proliferation requires MAPKs, which play important roles in the regulation of cellular responses during cell proliferation and stress. Among the 3 distinct MAPK families, p42/44 MAPKs are well known to be regulated by mitogens [17]. Our in vitro experiments with MECs demonstrated upregulation of p42/44 MAPK activity $24 \mathrm{~h}$ after puerarin treatment, which suggests that activation of the p42/44 MAPK pathway is associated with MECs proliferation and hence angiogenesis induced by puerarin.

In summary, puerarin possesses effects of antihypertension and stroke prevention by improving microcirculation in SHRs, which results from the increase in cerebral blood perfusion both by arteriole relaxation and p42/44 MAPKsmediated angiogenesis.

\section{Abbreviations}

SHRs:

MECs:

Spontaneously hypertensive rats

WKY:

Microvascular endothelial cells

PMSF:

MAP: Wistar-Kyoto

BDM: Phenylmethylsulfonyl fluoride

FBS: Mean arterial pressure

ECGS: 2,3-Butanedione monoxide

p42/44 MAPKs: p42/44 mitogen-activated protein kinases.

\section{Conflict of Interests}

The authors declare that there is no conflict of interests regarding the publication of this paper.

\section{Authors' Contribution}

Xu-Dong Wu and Chen Wang contributed equally to the paper.

\section{Acknowledgments}

This work was supported by the National Natural Science Foundation of China (nos. 81070186 and 81070130).

\section{References}

[1] A. C. Shore and J. E. Tooke, "Microvascular function in human essential hypertension," Journal of Hypertension, vol. 12, no. 7, pp. 717-728, 1994.

[2] T. F. T. Antonios, D. R. J. Singer, N. D. Markandu, P. S. Mortimer, and G. A. Mac-Gregor, "Structural skin capillary rarefaction in essential hypertension," Hypertension, vol. 33, no. 4, pp. $998-$ 1001, 1999.

[3] D. Versari, A. Virdis, L. Ghiadoni et al., "Effect of verapamil, trandolapril and their combination on vascular function and structure in essential hypertensive patients," Atherosclerosis, vol. 205, no. 1, pp. 214-220, 2009.

[4] K. H. Wong, G. Q. Li, K. M. Li, V. Razmovski-Naumovski, and K. Chan, "Kudzu root: traditional uses and potential medicinal benefits in diabetes and cardiovascular diseases," Journal of Ethnopharmacology, vol. 134, no. 3, pp. 584-607, 2011.

[5] S. Shibata, T. Murakami, Y. Nishikawa, and M. Harada, "The constituents of Pueraria root," Chemical Pharmaceutical Bulletin, vol. 7, no. 1, pp. 134-136, 1959.

[6] C. Zhao, H.-Y. Chan, D. Yuan, Y. Liang, T.-Y. Lau, and F.-T. Chau, "Rapid simultaneous determination of major isoflavones of Pueraria lobata and discriminative analysis of its geographical origins by principal component analysis," Phytochemical Analysis, vol. 22, no. 6, pp. 503-508, 2011.

[7] J.-H. Zhu, X.-X. Wang, J.-Z. Chen et al., "Effects of puerarin on number and activity of endothelial progenitor cells from peripheral blood," Acta Pharmacologica Sinica, vol. 25, no. 8, pp. 1045-1051, 2004.

[8] W. Wang, H. D. Zhang, J. Zhu, P. H. Wang, R. C. Sun, and Q. Deng, "Effects of puerarin on stroke in stroke-prone spontaneously hypertensive rats," Chinese Journal of Hypertension, vol. 8, pp. 58-61, 2008 (Chinese).

[9] X. H. Liu, F. Y. Liu, L. R. Cai, S. Sun, N. Tian, and C. S. Tang, "Effects of human urotensin II on pia mater microcirculation in rats," Chinese Journal of Applied Physiology, vol. 20, no. 1, pp. 46-49, 2004.

[10] A. C. Conti, R. Raghupathi, J. Q. Trojanowski, and T. K. McIntosh, "Experimental brain injury induces regionally distinct apoptosis during the acute and delayed post-traumatic period," The Journal of Neuroscience, vol. 18, no. 15, pp. 5663-5672, 1998.

[11] H. Parfenova, T. H. Eidson, and C. W. Leffler, "Upregulation of COX-2 in cerebral microvascular endothelial cells by smooth muscle cell signals," The American Journal of Physiology: Cell Physiology, vol. 273, no. 1, pp. C277-C288, 1997.

[12] S. Gillis, R. Kozak, M. Durante, and M. E. Weksler, "Immunological studies of aging. Decreased production of and response to T cell growth factor by lymphocytes from aged humans," The Journal of Clinical Investigation, vol. 67, no. 4, pp. 937-942, 1981.

[13] X. H. Liu, F. Grund, G. K. Kanellopoulos, K. Kvernebo, and A. Ilebekk, "Myocardial extracellular signal regulatory kinases are activated by laser treatment," The Journal of Cardiovascular Surgery, vol. 44, no. 1, pp. 1-7, 2003.

[14] X.-P. Song, P.-P. Chen, and X.-S. Chai, "Effects of puerarin on blood pressure and plasma renin activity in spontaneously hypertensive rats," Acta Pharmacologica Sinica, vol. 9, no. 1, pp. 55-58, 1988 (Chinese).

[15] X. Xu and X. Zheng, "Potential involvement of calcium and nitric oxide in protective effects of puerarin on oxygenglucose deprivation in cultured hippocampal neurons," Journal of Ethnopharmacology, vol. 113, no. 3, pp. 421-426, 2007.

[16] L. Chen, Q. Chai, A. Zhao, and X. Chai, "Effect of puerarin on cerebral blood flow in dogs," Zhongguo Zhong Yao Za Zhi, vol. 20, no. 9, pp. 560-562, 1995 (Chinese).

[17] C. Page and A. F. Doubell, "Mitogen-activated protein kinase (MAPK) in cardiac tissues," Molecular and Cellular Biochemistry, vol. 157, no. 1-2, pp. 49-57, 1996. 


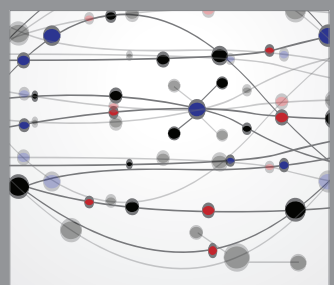

The Scientific World Journal
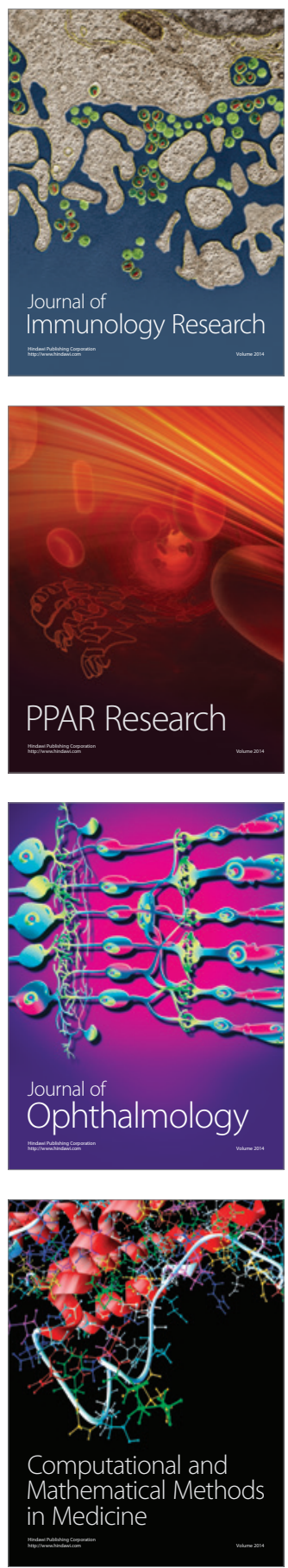

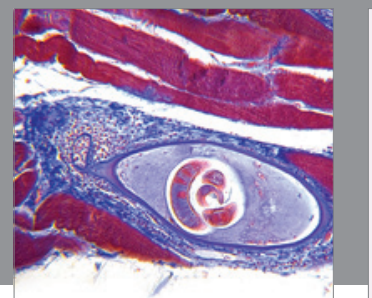

Gastroenterology

Research and Practice
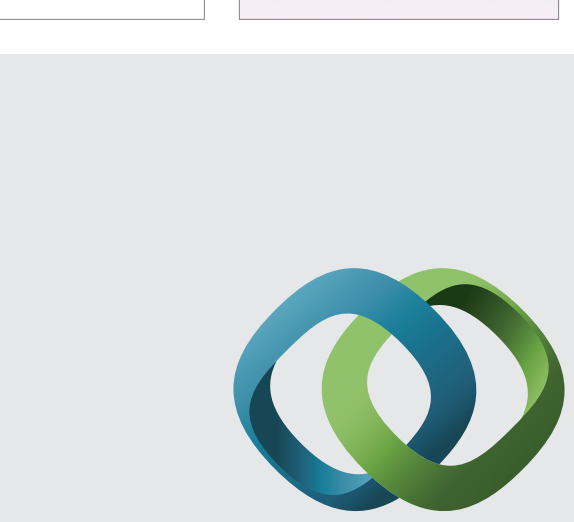

\section{Hindawi}

Submit your manuscripts at

http://www.hindawi.com
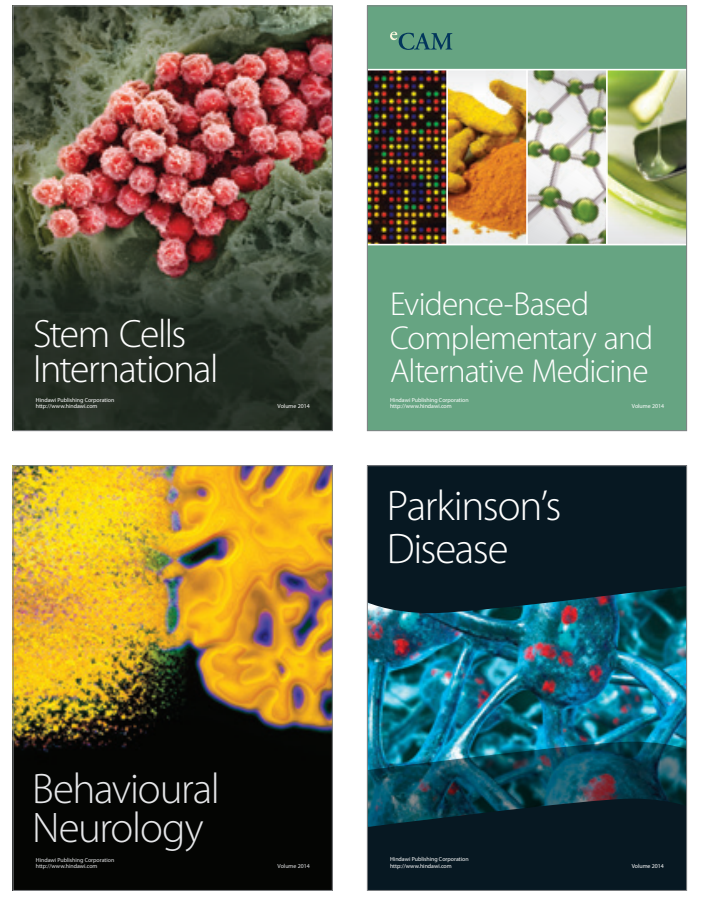
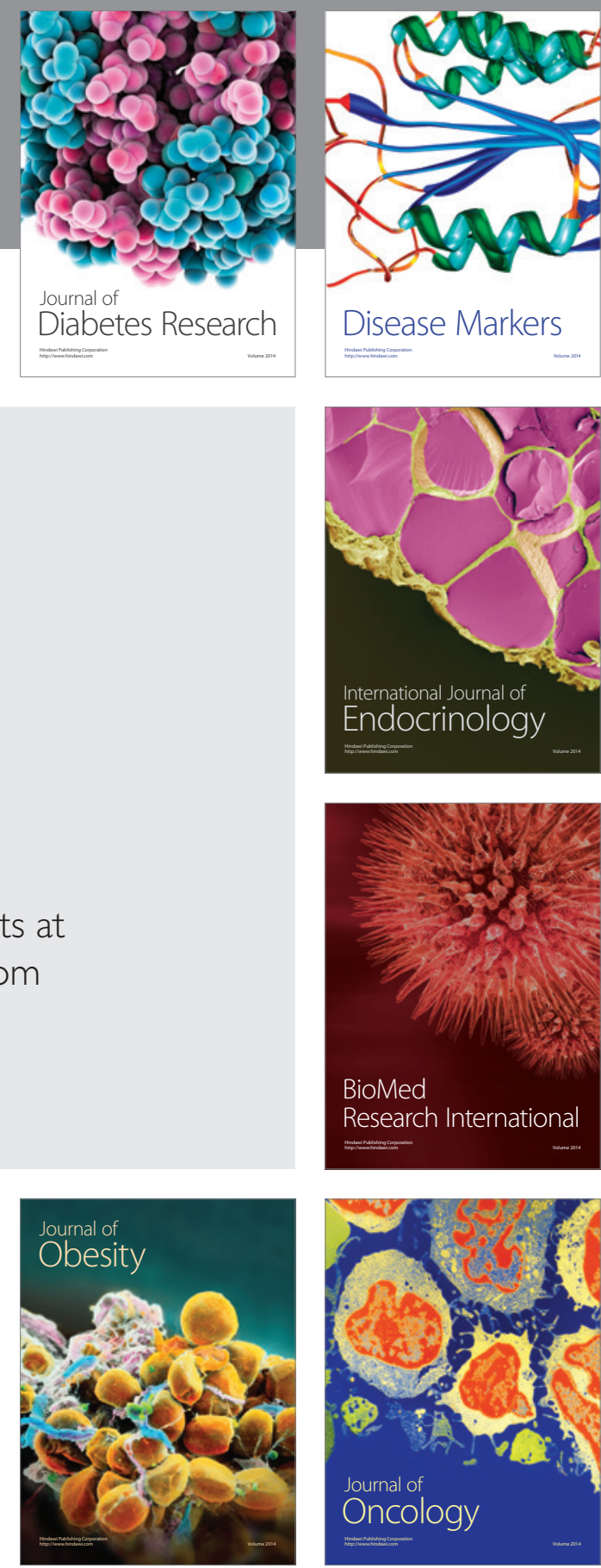

Disease Markers
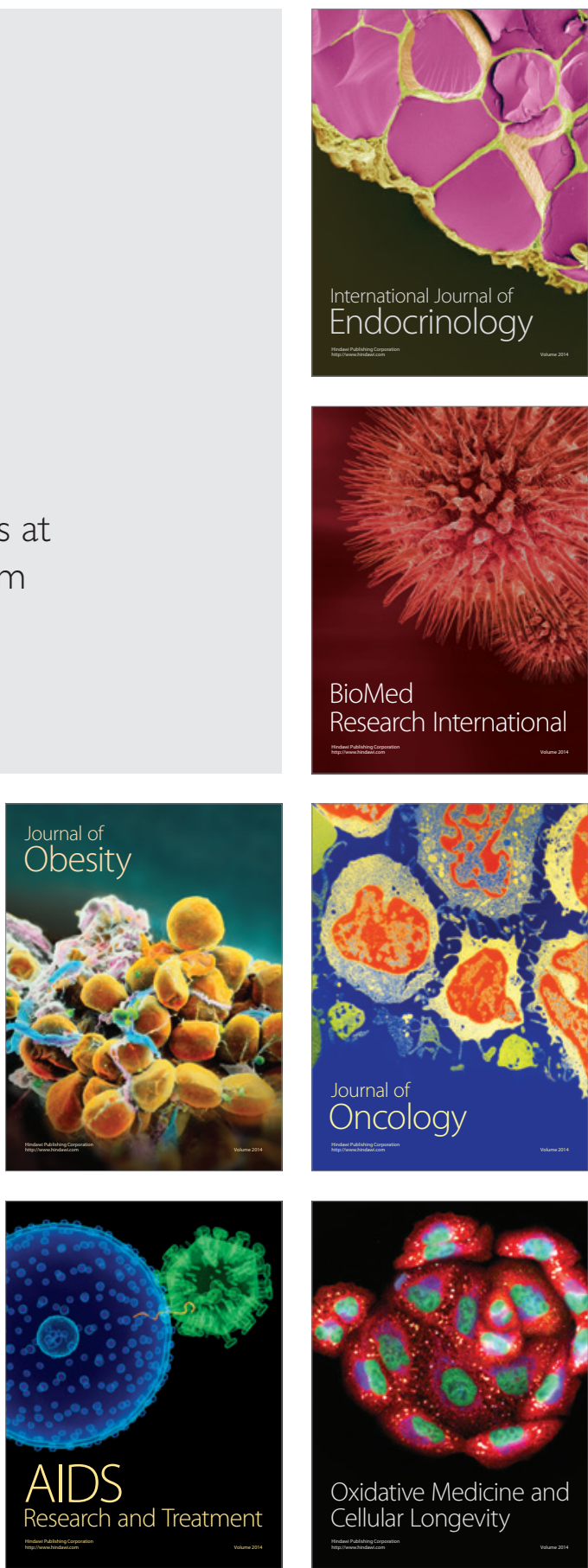